\section{Annual General Meeting: Technical Management Project Committee Report}

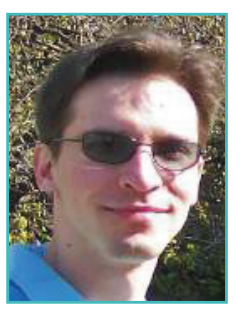

\section{George Vasilios Magklaras}

University of Oslo, The Biotechnology Centre of Oslo, Norway

Received 23 July 2013; Published 19 August 2013

The Technical Management Project Committee (TM PC) performed the following tasks for the period 2012-2013:

- specified and implemented a cloud virtualised host server, to transfer the main Web and EMBnet node journal operations. The Linode server provider was chosen;

- completed the transfer of the following domains from the Norwegian EMBnet node servers to the Linode hosting provider, minimising the impact on users and maintaining availabi-lity of operations:

o http://www.embnet.org

- http://conference.embnet.org

- helped the new website team to resolve a number of system issues in the deployment process of the new EMBnet website;

- set up a newly configured Adobe Connect Video and Audio Meeting software suite, in an attempt to help the EMBnet Operational Board (OB) choose an alternative way to conduct virtual general meetings (VGMs). The server ope-rates at the following URL: https:/l connect.uninett.no/testembnet

Currently the TM PC continues to manage:

- the EMBnet.journal Web server', and is helping the Swedish EMBnet node to transfer its content to a new server in Sweden;

- the EMBnet mailing lists

- the EMBnet DNS operations

- Because the TM PC Chair could not attend the AGM, new committee members willing to collaborate in the activity of the TM PC were hired after the AGM. The Committee is now composed of:George Magklaras - Chair and Secretary (NO), Gang Cheng - Member (NO), RomualdoZayas Lagunas - Member (MX)

The TM PC chair wishes to thank all the previous TM PC members for their contribution and effort. The following goals of the TMPC are set for the remaining activity time until the next AGM (2014):

a. improve/run the Adobe Connect Video and Audio meeting software for EMBnet;

b. manage the transition of EMBnet.journal to a new server, owing to decommissioning of the Norwegian EMBnet node server proteas.uio. no;

c. manage the transitioning of the EMBnet node DNS from Sweden to Linode and

d. secondary DNS servers so that continuity of operations is sustained;

e. manage the transitioning of the EMBnet node mailing list from Sweden to Linode and other servers, to ensure that communications are not disrupted.

The TMPC will meet twice every month, at a predetermined date and time, as called by the TM PC Chair, using the Adobe Connect Video and Audio Meeting software suite service. All members are expected to attend these meetings and contribute towards the specified goals/tasks above. 